4. Raghunathan V, Dhaliwal MS. Pharmacological management of COVID-19. J Pediatr Crit Care. 2020;7: S42-8.

5. Horby P, Lim WS, Emberson JR, et al. Dexamethasone in hospitalized patients with COVID-19 - Preliminary Report. N Engl J Med. 2020; NEJMoa2021436. [Epub ahead of print].

6. Barsoum Z. Pediatric asthma and coronavirus (COVID19)-Clinical presentation in an asthmatic child-SN Compr Clin Med. 2020;1-3. [Epub ahead of print].
7. Amirav I, Newhouse MT. Transmission of coronavirus by nebulizer: A serious, underappreciated risk. CMAJ. 2020; 192:E346.

8. COVID-19: GINA Answers to Frequently Asked Questions on Asthma Management - Global Initiative for Asthma - GINA. Global Initiative for Asthma - GINA. 2020. Accessed September 16, 2020. Available from: https:/ /ginasthma.org/covid-19-gina-answers-to-frequentlyaskedquestions-on-asthma-management.

\section{Neurological Manifestations of COVID-19 in Children}

Coronavirus disease 2019 (COVID-19) in children is mostly an asymptomatic or mildly symptomatic infection [1]. We seldom suspect COVID-19 in children with non-respiratory complaints, more so with isolated neurological manifestations. we present our experience of treating three children of COVID19 who presented with only neurological symptoms.

A 2-year-old previously healthy boy who had one day fever, three watery stools and pain abdomen, presented with febrile status epilepticus, hypotensive shock and hypoxia. A diagnosis of acute febrile encephalopathy was entertained and he was started on fluid resuscitation. He was shifted to critical care unit where he was mechanically ventilated in view of poor respiratory efforts with encephalopathy and received ceftriaxone, vancomycin and acyclovir. Reverse transcription polymerase chain reaction (RT-PCR) for SARS-CoV-2 (severe acute respiratory syndrome coronavirus 2 ) on a nasopharyngeal swab was positive and his antibody testing in serum was negative. His cerebrospinal fluid (CSF) analysis was in normal limits with negative RT-PCR for SARS-CoV-2. He fulfilled the criteria for multisystem inflammation syndrome MIS-C in children and was treated with intravenous immunoglobulin (IVIG) 2 grams $/ \mathrm{kg}$ along with remdesivir. His fever and requirement for inotrope support persisted, and intravenous methylprednisolone $(10 \mathrm{mg} / \mathrm{kg} /$ day $)$ was given for 3 days. His general condition improved, he did not have any further seizures, and was extubated after 48 hours. He was switched over to oral prednisolone for 2 weeks and low dose aspirin for 6 weeks, and was doing well on follow-up six weeks later.

A 15-month-old previously healthy boy presented with simple febrile seizures. On day two, he developed a maculopapular rash over the extremities with bilateral non-purulent conjunctival congestion, periorbital puffiness and cheilitis. He had persistent high-grade fever of $>103^{\circ} \mathrm{F}$ even on the fifth day and was referred for further management. His father had confirmed SARS-CoV-2 infection one month back. He fulfilled the criteria for MIS-C with Kawasaki disease phenotype, and was treated with intravenous immunoglobulin ( $2 \mathrm{~g} / \mathrm{kg})$, aspirin and steroids, as in the previous child. He was well on follow-up four weeks later.
An 8-month-old boy was brought with complaints of highgrade fever of $103^{\circ} \mathrm{F}$ for one day, followed by first episode of generalized tonic-clonic seizure lasting for more than 20 minutes on day one of illness. He was given intravenous midazolam followed by intravenous levetiracetam as the seizure episode was prolonged. There was a history of contact with confirmed SARS-CoV-2 in a close relative. His RT-PCR for SARS-CoV-2 in nasopharyngeal swab was positive. As the child did not have any encephalopathy or meningeal signs and no further episodes of seizures, CSF analysis and neuroimaging were deferred. He became afebrile from day three of illness. He was discharged on oral levetiracetam with a diagnosis of febrile status epilepticus, and is well on 2-weeks follow up.

With increasing numbers of SARS-CoV-2 infections, nonrespiratory manifestations are being reported across all age groups. The reason hypothesized is the distribution of angiotensin-converting enzyme 2 receptors (ACE-2R) or unexplained immune mechanism. ACE-2R are also present on the endothelial cells in the cerebral vasculature. Neurological manifestations in COVID-19 can be due to virus breaching the blood-brain barrier and entering the brain either trans-neuronally via the olfactory mucosa that has a relatively high expression of the ACE2 receptors, which then through olfactory nerve, crosses the cribriform plate or via hematogenous route [1] or as sepsis-induced coagulopathy leading to cerebral infarction [2] or immune-mediated neurological syndrome or can travel retrogradely via axonal transport to the brain from the gut or lungs. Few autopsy studies have demonstrated the presence of the virus in capillary endothelial cells of the frontal lobe of the brain [3]. The virus can also reach the brain by trojan horse mechanism via infected leukocytes migration across the blood brain barrier [4].

Seizures, encephalopathy, agitation, diffuse upper motor neuron signs, encephalitis, acute necrotizing encephalopathy, stroke, anosmia, ageusia, and Guillain-Barré syndrome have all been reported in adults with COVID-19 [5]. Encephalopathy (diffuse brain dysfunction) and encephalitis (acute, diffuse, inflammatory condition of the brain) are a major devastating presentation. Intense inflammatory response against the virus, triggers cytokine storm causing subsequent hypoxic and metabolic insults resulting in multiple organ failure including diffuse brain dysfunction. Altered consciousness is the hallmark clinical feature of encephalopathy. Individuals with encephalopathy/encephalitis are either severely or critically ill 
and have a poor prognosis [4]. In a case series of four children under 18 years of age who presented with severe COVID-19 infection, the neurological symptoms included encephalopathy, headache, brainstem, cerebellar signs, muscle weakness, and reduced reflexes. MRI brain had signal changes in the splenium of the corpus callosum in all four patients and T2-hyperintense lesions associated with restricted diffusion were seen in three children [6]. In a recent multi-centric retrospective study which analyzed the MRI findings in adults with severe COVID 19 infection, signal abnormalities located in the medial temporal lobe, non-confluent multifocal white matter hyperintense lesions on FLAIR and diffusion with variable enhancement, associated with hemorrhagic lesions, and (c) Extensive and isolated white matter microhemorrhages were the most common findings. The presence of hemorrhage was frequent, and the detection is of clinical importance as it was associated with worse respiratory, neurological, and biological status [7].

Internationally accepted case definitions for MIS-C are still evolving. In our case series, all were confirmed cases of COVID19 , of which the first two children had neurological manifestations of acute febrile encephalopathy and febrile seizure with features of MIS-C while the third child presented as febrile status epilepticus. One child with MIS-C had fulfilled the criteria for incomplete Kawasaki phenotype (fever $>5$ days, rash, bilateral non-purulent conjunctival congestion, cheilitis) according to AHA guidelines. Many MIS-C cases present as Kawasaki disease shock syndrome with Kawasaki-like clinical symptoms, cardiac impairment and shock [9].

There were a few limitations in our observations. Imaging studies and CSF analysis were not done in all. We speculate that COVID-19 being a respiratory virus, other systemic manifestations especially neurological presentations may go unrecognized. In this pandemic situation, any child with primary neurologic symptoms and fever, with mild or absent respiratory symptoms, it could either be a part of MIS-C or a self-limiting finding of pediatric COVID-19 infection.

Acknowledgements: Dr S Balasubramnain, Head, Department of
Pediatrics and Dr Bala Ramachandran, Head, Pediatric Intensive Care Unit, Kanchi Kamakoti CHILDS Trust hospital for editing the draft.

\author{
S LAKSHAN RAJ ${ }^{1 *}$ T VASANTHI, ${ }^{1}$ REVANTH BAINENI ${ }^{2}$ AND \\ Somu Sivabalan ${ }^{1}$ \\ Department of ${ }^{1}$ Pediatrics and ${ }^{2}$ \\ Pediatric Intensive Care Unit, \\ Kanchi Kamakoti CHILDS Trust Hospital, Chennai, India. \\ *doc.lakshanraj2311@gmail.com
}

\section{REFERENCES}

1. Natoli S, Oliveira V, Calabresi P, Maia LF, Pisani A. Does SARS-Cov-2 invade the brain? Translational lessons from animal models. Eur J Neurol. 2020;27: 1764-73.

2. Schupper AJ, Yaeger KA, Morgenstern PF. Neurological manifestations of pediatric multi-system inflammatory syndrome potentially associated with COVID-19. Childs Nerv Syst. 2020;36:1579-80.

3. Paniz-Mondolfi A, Bryce C, Grimes Z, et al. Central nervous system involvement by severe acute respiratory syndrome coronavirus -2 (SARS-CoV-2). J Med Virol. 2020;92:699-702.

4. Garg RK, Paliwal VK, Gupta A. Encephalopathy in patients with COVID 19: A review. J Med Virol. 2020;117.

5. Mao L, Jin H, Wang M, et al. Neurologic manifestations of hospitalized patients with coronavirus disease 2019 in Wuhan, China. JAMA Neurol. 2020;77:683-90.

6. Abdel-Mannan O, Eyre M, Löbel U, et al. Neurologic and radiographic findings associated with COVID-19 infection in children. JAMA Neurol. 2020;77:1440-45.

7. Kremer S, Lersy F, Sèze J de, et al. Brain MRI findings in severe COVID-19: A retrospective observational study. Radiology. 2020 June 16. [E-pub ahead of print]

8. Gamez-Gonzalez L B, Moribe-Quintero I, CisnerosCastolo M, et al. Kawasaki disease shock syndrome; Unique and severe subtype of Kawasaki disease. Pediatr Int. 2018; 60:781-190.

\section{SARS-CoV-2 Encephalitis in an Adolescent Girl}

Severe acute respiratory syndrome coronavirus 2 (SARS-CoV2) virus can affect both the central and peripheral nervous system, and SARS neurological manifestations have also been rarely reported in children. We herein report COVID-19 encephalitis in a 13-year-old girl, who presented with status epilepticus and altered sensorium and had complete resolution of neurological symptoms in 48 hours.

A 13-year-old girl presented with fever for 2 days associated with headache and an episode of generalized tonic clonic seizures on day 2 of fever lasting for more than 30 minutes. She was treated with intravenous lorazepam and phenytoin sodium and in view of persistence of seizures and altered sensorium, she was referred to our center for further care. There was no history of cough, vomiting, head trauma, rash or drug ingestion. She was first born to non-consanguineous parents and developmentally normal for age. There was no past or family history of seizures. On examination at admission, she was febrile $\left(100^{\circ} \mathrm{F}\right)$, irritable and had altered sensorium. She had brisk deep tendon reflexes with an extensor plantar response. There were no signs of meningeal irritation. Her pupils were equal and reacting to light. There was no papilledema or focal neurological deficits. Cardiopulmonary and abdomen examination were normal. Her investigations revealed normal white cell counts and negative CRP. Her serum electrolytes including calcium and magnesium, liver function tests were normal. MRI 